\title{
The Correlation between Proprotein Convertase Subtilisin / Kexin Type 9 (PCSK9) and Insulin Resistance and the Components of Atherogenic Lipoprotein Phenotype in Males with Central Obesity
}

\author{
Yusmiati ${ }^{1,2^{*}}$, Burhanuddin Bahar ${ }^{1}$, Andi Wijaya ${ }^{1,2}$ \\ 1Post Graduate Program in Clinical Biochemistry, Hassanudin University, Makassar, Indonesia \\ ${ }^{2}$ Prodia Clinical Laboratory, Jakarta, Indonesia
}

\section{Abstract}

B

ACKGROUND: Proprotein Convertase Subtilisin /Kexin Type 9 (PCSK9) promotes the degradation of LDL receptor in hepatocytes. In vitro studies have proven that insulin increases the expression of PCSK9. Insulin resistance, a common condition in central obesity is characterized by dyslipidemia called Atherogenic Lipoprotein Phenotype (ALP). This study aimed to investigate the correlation between insulin resistance (HOMA-IR) and PCSK9, and to analyze whether this condition is related to the development of ALP in central obesity.

METHODS: This is an observational study with crosssectional design. The subjects consisted of 62 male adults with central obesity, aged $30-60$ years old. ELISA was used to measure plasma PSCK9.

RESULTS: The mean plasma PCSK9 concentration of the samples was $283.7 \mathrm{ng} / \mathrm{mL}$. PCSK9 had positive linear correlation with HOMA-IR $\left(r=0.225^{*}, \mathrm{p}=0.045\right)$ and Apo $\mathrm{B}(\mathrm{r}=0.245, \mathrm{p}=0.055)$. After controlling of HOMA-IR, PCSK9 had positive linear correlation with triglycerides $\left(\mathrm{r}=0.352^{*}, \mathrm{p}=0.045\right)$. In population with HOMA-IR > 2, crosstabs analysis showed that PCSK9 had significant correlation with triglycerides and Apo B with an odd ratio of $6,125\left(\mathrm{r}=0,376^{*}, \mathrm{p}=0,037\right)$. Triglyceride showed significant negative correlation with HDL cholesterol and ratio of LDL/Apo B, but neither HOMA-IR nor PCSK9 did.
CONCLUSION: In males with central obesity, PCSK9 is one of the factors mediating the occurence of ALP in insulin resistance.

KEYWORDS: PCSK9, Insulin Resistance, Atherogenic Lipoprotein Phenotype.

\section{Introduction}

PCSK9, also previously known as NARC-1 (Neural Apoptosis Regulated Convertase 1), is a protease that regulates Low Density Lipoprotein receptor (LDLr), a plasma membrane glycoprotein that function to clear cholesterol rich-LDL particles from the circulation (1).

PCSK9 is a 692-amino acid protein, expressed mainly in the liver, intestine, and kidney (2). PCSK9 is synthesized in endoplasmic reticulum as a $72 \mathrm{kDa}$ zymogen. PCSK9 consisted of 3 known domains: N-terminal prodomain, catalytic domain and carboxyl-terminal domain with unknown function (1). After its synthesis, this molecule undergoes autocatalytic cleavage between the prodomain and catalytic domain. The $\mathrm{N}$-terminal prodomain remains binded to the protein and acts as a chaperone to transport PCSK9 out of the cell (2).

The molecular mechanism by which PCSK9 targets LDLris quite complex.PCSK9 is secreted, and subsequently requires LDLr-mediated internalization to inhibit LDLr expression. In the acidic environment of endosome, the 
affinity of PCSK9 for LDLr increases 150 fold. PCSK9 binds to the epidermal growth factor (EGF)-like repeat A of LDLr, a region important for recycling of LDLr from endosome to the plasma membrane. This binding results in the redistribution of LDLr to lysosome, instead of to plasma membrane, and thus reducing the amount of LDLr at the membrane (1).

Besides its function in degrading LDLr, PCSK9 has also been thought to play role in the secretion of Apolipoprotein B (Apo B).Areport by Ouguerram et al.has revealed that in two individuals with S127R heterozygote mutation of PCKS9, there were 3 fold increase of production of Apo B and Very Low Density Lipoprotein (VLDL) (3). However, in other study using primary hepatocytes from rats as well as in hepatoma, over-expression of PCSK9 did not show any increase in the production of Apo B (4).

Some reports have shown that insulin has an effect on the PCSK9 level. Costet et al. showed that hyperinsulinemia in primary hepatocytes increased PCSK9 mRNA by $4-5$ fold (5). A study by Baass et al. in 1700 children aged 9-16 years old also showed a correlation between PCSK9 and HOMA-IR and insulin (6). On the other hand, a study in 21 non-diabetic male subjects showed that PCSK9 did not correlate significantly with insulin or insulin resistance (7).

Insulin resistance has a strong correlation with central obesity. This condition is characterized by the increase of triglyceride and small dense Low Density Lipoprotein (LDL) cholesterol, decrease of High Density Lipoprotein (HDL) cholesterol, with normal or slightly increase of LDL cholesterol. This dyslipidemia is termed Atherogenic Lipoprotein Phenotype (ALP). Although all these components of ALP might be affected by insulin resistance, data have shown that the effect of insulin resistance in accumulation and over secretion of VLDL, Apo B, and triglyceride is the central cause. Insulin resistance increases the production and secretion of VLDL and triglyceride, which in turn decrease the level of HDL cholesterol and turned the production of smaller LDL (8).

The hypothesis of this study is because insulin affects the production of PCSK9 and PCSK9 plays role in the uptake of apoB-containing lipoprotein (VLDL, LDL), PCSK9 might be one of the mechanisms by which insulin resistance causes ALP.

\section{Methods}

This was an observational study with cross-sectional design. The sample number was calculated using the alpha value of 0.05 and $r=0.04$, from which the minimum number of samples was set at 62 . The study subjects comprised 62 males with central obesity (waist circumference $\geq 90$ $\mathrm{cm}$ ), aged 30-60 years old, and were recruited randomly from the community. All subjects agreed to participate in this study by signing an informed consent prior to blood sampling. No subjects had abnormal liver function test, smoked, or consumed statin or fenofibrate.

Insulin resistance was defined as HOMA-IR $>2$. In the analysis, the study subjects were divided into two groups: insulin resistance group (HOMA-IR $>2$ ) and non-insulin resistance group (HOMA-IR $\leq 2$ ). Small dense LDL was defined as the ratio of LDL cholesterol/Apo B.

Allbiochemicalmarkersmeasurementswereperformed at Prodia Clinical Laboratory. Serum glucose, ALT, AST, triglyceride, HDL cholesterol, LDL cholesterol, and Apo B were measured using Prodia Clinical Laboratory routine procedures by ADVIA 1800 instrument from Siemens. Insulin was measured by immunochemiluminescent method using Immulite 2000. PCSK9 was measured by ELISA using kit from RnDSystems (Minneapolis, USA).

\section{Results}

Table 1 presents the characteristics of the study subjects. PCSK9 level ranged from 114.77 to $534.57 \mathrm{ng} / \mathrm{mL}$.

Because HOMA-IR was not normally distributed, we transformed the data into logarithmic data (log HOMA-IR) to make them normally distributed.

Log HOMA-IR showed significant positive correlation with Apo B ( $\left.\mathrm{r}=0,317^{* *}, \mathrm{p}=<0,01\right)$, but showed insignificant correlation with other component of ALP. The crosstabulation analysis also showed no significant correlation between Log HOMA-IR and either components of ALP. 
Table 1. Basic characteristics of the study subjects

\begin{tabular}{lccccc}
\hline Variables & Mean \pm 2 SD & Min & Max & Median & p \\
\hline $\mathrm{n}=62$ & & & & & \\
Age (years) & $41.77 \pm 8.482$ & 30 & 60 & 40 & 0.350 \\
WC $(\mathrm{cm})$ & $98.02 \pm 6.056$ & 90 & 116 & 97 & 0.218 \\
BMI $\left(\mathrm{kg} / \mathrm{m}^{2}\right)$ & $28.21 \pm 3.096$ & 22.32 & 36.44 & 27.52 & 0.224 \\
Glucose $(\mathrm{mg} / \mathrm{dL})$ & $106.55 \pm 44.641$ & 75 & 295 & 95 & $0.000^{\star *}$ \\
Insulin $(\mu \mathrm{lU} / \mathrm{mL})$ & $11.27 \pm 9.307$ & $<2$ & 63.5 & 9.25 & 0.066 \\
HOMA-IR & $2.95 \pm 2.535$ & 0.49 & 13.48 & 2.04 & $0.030^{*}$ \\
LDL Cholesterol (mg/dL) & $137.58 \pm 37.203$ & 74 & 248 & 134 & 0.882 \\
Apo B (mg/dL) & $106.68 \pm 22.351$ & 65 & 180 & 403 & 0.887 \\
HDL Cholesterol (mg/dL) & $43.00 \pm 7.566$ & 23 & 63 & 43.5 & 0.900 \\
Triglyceride $(\mathrm{mg} / \mathrm{dL})$ & $146.39 \pm 67.405$ & 38 & 362 & 140.5 & 0.575 \\
Rasio of LDL/Apo B & $1.28 \pm 0.164$ & 0.72 & 1.51 & 1.31 & 0.224 \\
PCSK9 (ng/mL) & $283.7 \pm 92.430$ & 114.77 & 534.57 & 273.63 & 0.621 \\
\hline
\end{tabular}

p showing significance levels of Kolmogorov-smirnov test, ${ }^{\star \star \star \star \star}$ indicate data not normally distributed, BMI = Body Mass Index; HOMA-IR $=$ Homeostasis Model Assessment-Insulin Resistance, LDL = Low Density Lipoprotein, Apo B = Apolipoprotein B, HDL = High Density Lipoprotein, PCSK9 = Proprotein Convertase Subtilisin Kexin type 9.

From all variables, PCSK9 showed significant positive correlation with insulin $\left(\mathrm{r}=0.325^{*}, \mathrm{p}<0.05\right)$ and HOMA-IR $\left(\mathrm{r}=0.225^{*}, \mathrm{p}<0.05\right)$. PCSK9 did not show any significant correlation with triglyceride $(\mathrm{r}=0.167, \mathrm{p}$ $=0.193)$, Apo B $(\mathrm{r}=0.245, \mathrm{p}=0.055), \mathrm{HDL}$ cholesterol $(\mathrm{r}=0.089, \mathrm{p}=0.490)$, LDL cholesterol $(\mathrm{r}=0,156, \mathrm{p}=$
$0,223)$ or ratio of LDL/Apo B $(r=-0.112, p=0.386)$. After controlling for HOMA-IR, PCSK9 showed significant correlation with triglyceride $(\mathrm{r}=0.382 *, \mathrm{p}=0.045)$.

Figure 1 shows the scattergram of correlation between PCSK9 and insulin and log HOMA-IR.

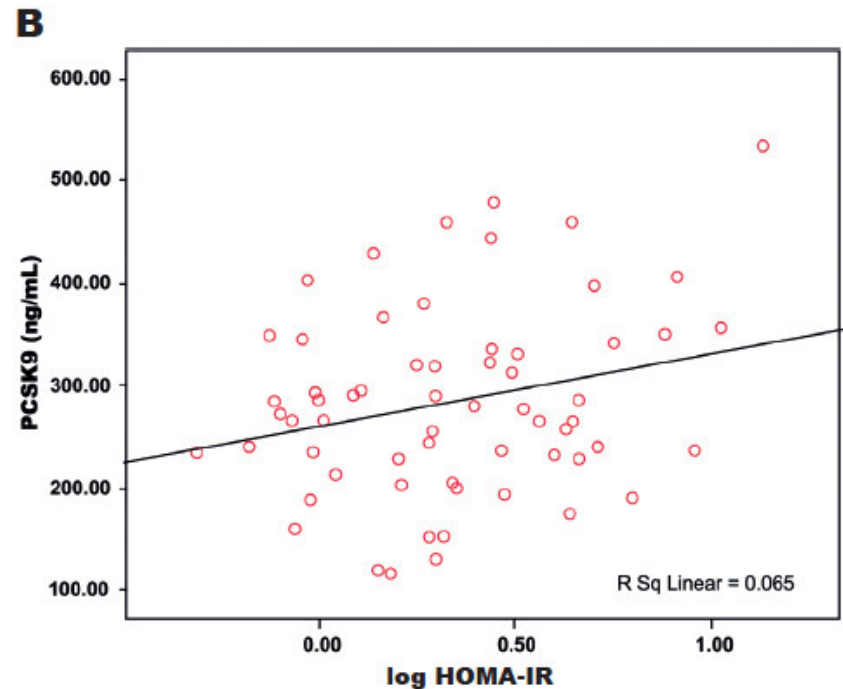

Figure 1. Scattergram of PCSK9 and Insulin (A) and Log HOMA-IR (B). 
A

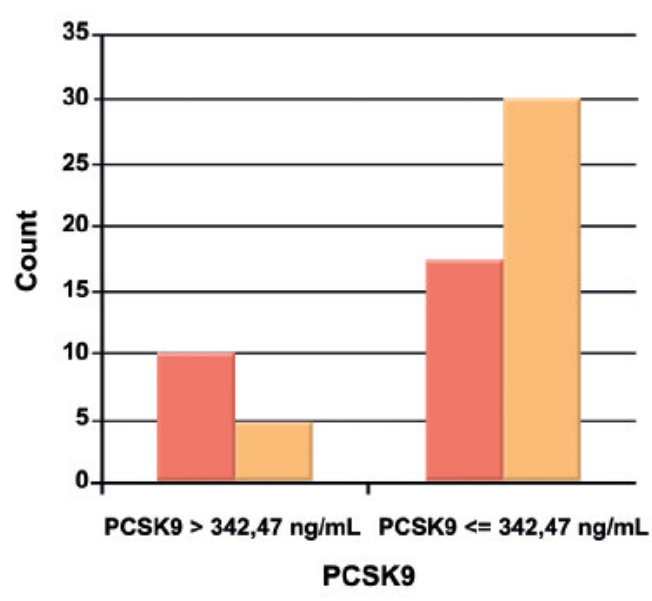

B

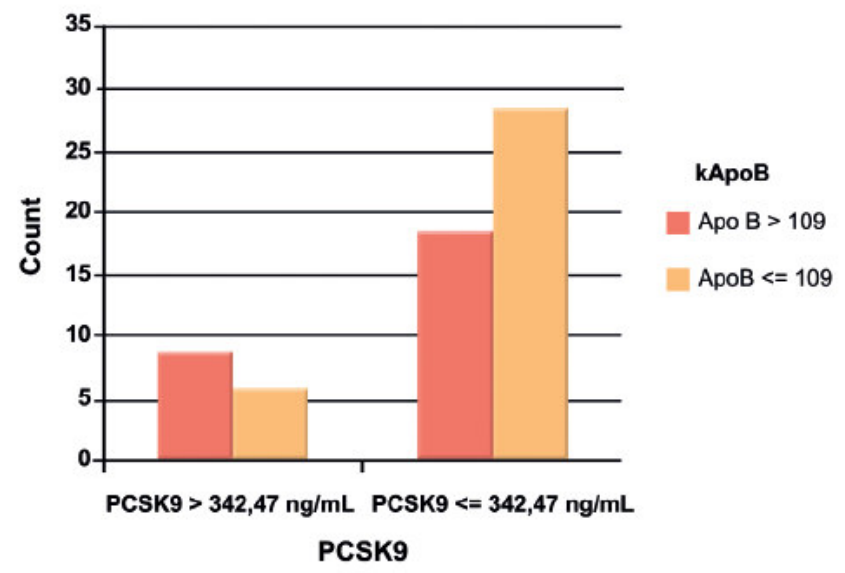

Figure 2. The crosstabulation analysis between PCSK9 and Triglyceride (A) and Apo B (B)

From the crosstabulation analysis we found that PCSK9 showed significant positive correlation ( $\mathrm{p}$ $<0.05$ ) with Triglyceride with an odd ratio of 3.529 , but insignificant correlation with other component of ALP, although in PCSK9 $>75^{\text {th }}$ percentile, the odd ratio for Apo B $>109 \mathrm{mg} / \mathrm{dL}$ was almost 2.5 times.

When we grouped the subjects into two groups based on their HOMA-IR value and analyzed the correlation of PCSK9 with the components of ALP, we found that in the group with HOMA-IR $>2$, PCSK9 showed significant correlation with triglyceride and Apo B $\left(r=0,376^{*}, \mathrm{p}=\right.$ $0,037)$ with the odd ratio of $6 \cdot 125$. These correlation were not found in the group with HOMA-IR $\leq 2$.

Within the components of ALP, Triglyceride showed significant correlation with Apo B $\left(r=0.365^{*}, \mathrm{p}<0.01\right)$, HDL Cholesterol $\left(r=-0.256^{*}, p<0.05\right)$, and ratio of LDL/ Apo B $\left(r=-0.504^{* *}, p<0.000\right)$. No significant correlation was found between PCSK9 and LDL cholesterol $(r=0.156$, $\mathrm{p}=0.223$ ).

\section{Discussion}

In this study we presented the data of PCSK9 level in central obese males and analyzed its correlation to insulin resistance and atherogenic lipoprotein phenotype. PCSK9 level reported from this study was relatively low (114.77-534.57 $\mathrm{ng} / \mathrm{mL}$ ) compared to other publication. Lakoski reported a mean of 33-2988 ng/mL (9), Lambert et al. reported from 115 diabetic patients PCSK9 level ranging from 100-9300 $\mathrm{ng} / \mathrm{mL}$ (10), whereas Horton in his review article stated that PCSK9 from various publications ranged from 500-4000 $\mathrm{ng} / \mathrm{mL}$ (2). The difference might be attributed to several factors, one of which is the measurement method. Most studies measured PCSK9 by ELISA method developed in their own labs, some using monoclonal antibody, which showed lower level and some using polyclonal antibody, which showed higher level. This report measured PCSK9 using an ELISA kit using monoclonal antibody to capture and polyclonal antibody to detect. Another factor might be the difference in the subjects of the study. This study was done on Asian race, whereas other reports mostly studied on the Caucasian or African race.

We found significant correlation between PCSK9 and insulin and HOMA-IR. Interestingly, one subject with the highest insulin level in this population $(63.5 \mathrm{mIU} / \mathrm{mL})$ also had the highest PCSK9 level $(534.57 \mathrm{ng} / \mathrm{mL})$ as well as the highest HOMA-IR (13.48). When this subject was excluded from the data, the correlation between PCSK9 and insulin and HOMA-IR became statistically insignificant.

The scattergram also showed a blank area between other samples and the extreme samples. There might be some class of population that was not captured in this study that could fill in that blank area.

The correlation between insulin and PCSK9 has been shown by some reports. Baass et al. reported that with independence from age, glucose, and adiponectin, insulin positively correlated with PCSK9. Previous reports in animals also showed that in diabetes type 1- induced rats there was a significant decrease in LDLr mRNA, where as the LDLr protein left unaffected. The explanation of this is that PCSK9 level also decreased in hypoinsulinemia 
rats, thus the degradation of LDLr protein was reduced. It was also found that in hyperinsulinemia rats, the PCSK9 mRNA was increased (6).

The correlation between PCSK9 and HOMA-IR has been inconsistent in several publications. Chan et al. in 2009 reported that in 21 non-smoking males, PCSK9 did not correlate with HOMA-IR or insulin (7), whereas Baass et al.reported that in 1739 French children and adolescents, PCSK9 correlated significantly with HOMA-IR, glucose, and insulin (6).

Our study also found significant correlation between PCSK9 with insulin and HOMA-IR, suggesting that PCSK9 level in central obese men was higher than in non-obese men. We measured that level of PCSK9 in 15 non-obese male (data not shown), and found no significant difference in PCSK9 level between obese and non-obese males. The explanation might be that there were only small samples of non-obese subjects observed, but unfortunately, due to the limitation of the PCSK9 reagents, we could not assess more samples of non-obese males.

Using Pearson test, we found that PCSK9 had insignificant correlation with any components of ALP. In the cross-tabulation analysis, PCSK9 showed significant correlation with triglyceride with the odd ratio of 3.5 ( $\mathrm{p}=$ 0.044). In other words, in a population with PCSK9 level above $75^{\text {th }}$ percentile, there was 3.5 times risk of having abnormal triglyceride level.

After controlling on HOMA-IR, we found significant correlation between PCSK9 and triglyceride. In the insulin resistance group, we also found significant correlation between PCSK9 and triglyceride and Apo B.

In insulin resistance state, there was an increase in the triglyceride in the liver that acted as a source for VLDL secretion and there was also an increase in the secretion of Apo B. This would drive an increase in the production of triglyceride rich-VLDL, thus the triglyceride in the circulation would increase. The significant correlation of PCSK9 with triglyceride after controlling on HOMA-IR indicated that in insulin resistance state, PCSK9 would increase the triglyceride level even more.

This was also shown in crosstabulation analysis. In the groups with HOMA-IR >2, PCSK9 showed significant correlation with triglyceride and Apo B, with an odd ratio of 6.125. In other words, individuals with PCSK9 level above $75^{\text {th }}$ percentile $(342.47 \mathrm{ng} / \mathrm{mL})$ would have 6 times higher risk for having abnormal level of triglyceride and Apo B. Thus PCSK9 is one factor that plays role in the development of ALP in insulin resistance by affecting the triglyceride and Apo B level, which in turn will decrease HDL cholesterol and shift the LDL production to smaller and denser LDL.
One interesting finding in this study was that there was no significant correlation between PCSK9 and LDL cholesterol $(\mathrm{r}=0.156, \mathrm{p}=0.223)$, whereas almost all publications on PCSK9 showed significant correlation. When we looked at the individual data, there was a patient having high PCSK9 level (479.17 ng/mL), but quite low level of LDL cholesterol $(74 \mathrm{ng} / \mathrm{mL})$. If we excluded this patient, the correlation would be stronger $(\mathrm{r}=0.224, \mathrm{p}=$ 0.083 ) although still statistically insignificant. We assumed there were other factors (metabolic, genetic, etc) in some patients that altered the correlation between PCSK9 and LDL cholesterol. Besides, unlike other studies, this study focused only on central obese subjects. Another factor that might contribute to this discrepancy is that other studies were conducted either on Caucasian or African race, whereas this study was done on Asian race. Larger studies with more variant subjects are needed to better understand the correlation between PCSK9 and LDL cholesterol and the factors affecting it.

\section{Conclusions}

In males with central obesity, PCSK9 is one of the factors mediating the occurrence of ALP in insulin resistance by affecting triglyceride and Apo B.

\section{Acknowledgements:}

We thank Prodia Education and Research Institute for funding this research, Prodia Reference Lab and Prodia Research and Esoteric Test Lab for their assistance in the biomarkers testing.

\section{References:}

1. Peterson AS, Fong LG, Young SG. PCSK9 Function and Physiology. J. Lipid Res 2008; 49:1152-6

2. Horton JD, Cohen JC, Hobbs HH. PCSK9: A Convertase That Coordinates LDL Catabolism. J Lipid Res 2009: S172-7.

3. Ouguerram K, Chetiveaux M, Zair Y, Costet P, Abifadel M, Varret $\mathrm{M}$, et al. Apolipoprotein B100 Metabolism in AutosomalDominant Hypercholesterolemia Related to Mutations in PCSK9. Arterioscler Thromb Vasc Biol. 2004; 24: 1448-53

4. Maxwell KN and Breslow JL. Proprotein Convertase Subtilisin Kexin 9: The Third Locus Implicated in Autosomal Dominant Hypercholesterolemia. Curr Opin Lipidol. 2005; 16: 16772.

5. Costet $P$, Cariou B, Lambert G, Lalanne F, Lardeux B, Jarnoux $\mathrm{AL}$, et al. Hepatic PCSK9 Expression Is Regulated by Nutritional Status via Insulin and Sterol Regulatory ElementBinding Protein 1c. J Biol Chem. 2006; 281: 6211-8. 
6. Baass A, Dubuc G,Tremblay M, Delvin EE, O'Loughlin J, Levy $\mathrm{E}$, et al. Plasma PCSK9 Is Associated with Age, Sex, and Multiple Metabolic Markers in A Population-Based Sample of Children And Adolescents. Clin Chem. 2009; 55: 163745.

7. Chan DC, Lambert G, Hugh P, Barrett B, Rye KA, Ooi E, et al. Plasma Proprotein Convertase Subtilisin/ Kexin Type 9: A Marker of LDL Apolipoprotein B-100 Catabolism? Clin Chem. 2009; 55: 2049-52
8. Ginsberg HL, Zhang YL, Hernandez-Ono A. Metabolic Sydrome: Focus on Dislipidemia. Obesity 2006; 14 (Supp.): 41S-9S

9. Lakoski SG, Lagace TA, Cohen JC, Horton JD, Hobbs HH. Genetic and Metabolic Determinants of Plasma PCSK9 Levels. J Clin Endrocrinol Metab 2009; 94: 2537-43

10. Lambert G, Ancellin N, Charlton F, Comas D, Pilot J, Keech A, et al. Plasma PCSK9 Concentrations Correlate with LDL and Total Cholesterol in Diabetic Patients And are Decreased by Fenofibrate Treatment. Clin Chem 2008; 56: 1038-45. 